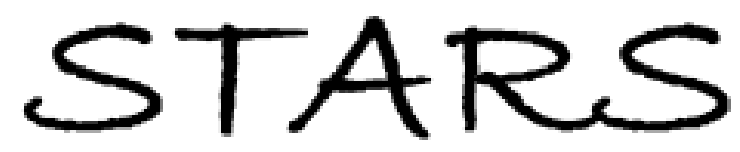

University of Central Florida

STARS

$1-1-2013$

\title{
Nanoparticle agglomeration in an evaporating levitated droplet for different acoustic amplitudes
}

\author{
Erick Tijerino \\ University of Central Florida \\ Saptarshi Basu \\ Ranganathan Kumar
}

Find similar works at: https://stars.library.ucf.edu/facultybib2010

University of Central Florida Libraries http://library.ucf.edu

This Article is brought to you for free and open access by the Faculty Bibliography at STARS. It has been accepted for inclusion in Faculty Bibliography 2010 s by an authorized administrator of STARS. For more information, please contactSTARS@ucf.edu.

\section{Recommended Citation}

Tijerino, Erick; Basu, Saptarshi; and Kumar, Ranganathan, "Nanoparticle agglomeration in an evaporating levitated droplet for different acoustic amplitudes" (2013). Faculty Bibliography 2010s. 4759.

https://stars.library.ucf.edu/facultybib2010/4759

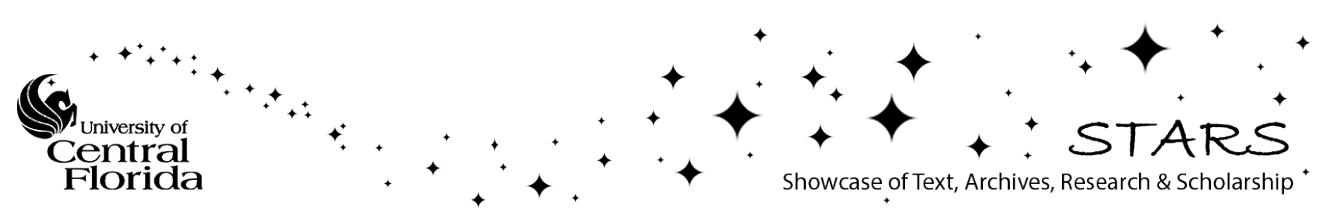




\section{Nanoparticle agglomeration in an evaporating levitated droplet for different acoustic amplitudes}

Cite as: J. Appl. Phys. 113, 034307 (2013); https://doi.org/10.1063/1.4775791

Submitted: 09 August 2012 . Accepted: 21 December 2012 . Published Online: 17 January 2013

Erick Tijerino, Saptarshi Basu, and Ranganathan Kumar
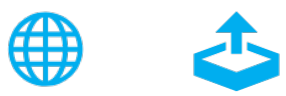

View Online

\section{ARTICLES YOU MAY BE INTERESTED IN}

Insight into morphology changes of nanoparticle laden droplets in acoustic field Applied Physics Letters 102, 141602 (2013); https://doi.org/10.1063/1.4801502

Drying of acoustically levitated droplets of liquid-solid suspensions: Evaporation and crust formation

Physics of Fluids 14, 2289 (2002); https://doi.org/10.1063/1.1483308

Structural morphology of acoustically levitated and heated nanosilica droplet

Applied Physics Letters 97, 123106 (2010); https://doi.org/10.1063/1.3493178

\section{Applied Physics Reviews} Now accepting original research

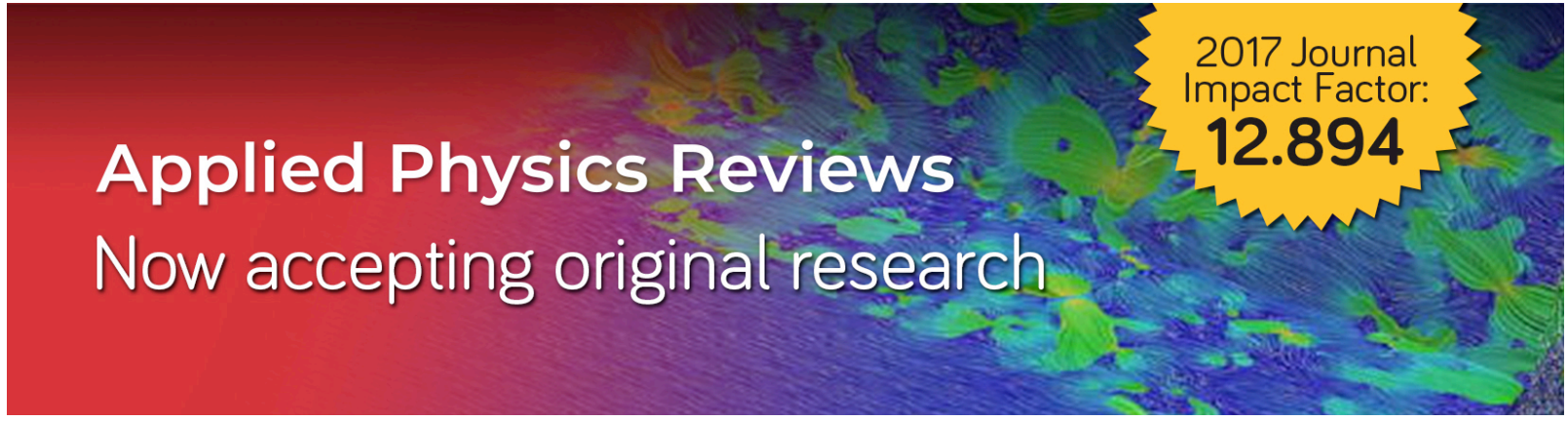




\title{
Nanoparticle agglomeration in an evaporating levitated droplet for different acoustic amplitudes
}

\author{
Erick Tijerino, ${ }^{1, a)}$ Saptarshi Basu, ${ }^{2, a)}$ and Ranganathan Kumar ${ }^{1, a), b)}$ \\ ${ }^{1}$ University of Central Florida, Orlando, Florida 32816, USA \\ ${ }^{2}$ Indian Institute of Science, Bangalore, India
}

(Received 9 August 2012; accepted 21 December 2012; published online 17 January 2013)

\begin{abstract}
Radiatively heated levitated functional droplets with nanosilica suspensions exhibit three distinct stages namely pure evaporation, agglomeration, and finally structure formation. The temporal history of the droplet surface temperature shows two inflection points. One inflection point corresponds to a local maximum and demarcates the end of transient heating of the droplet and domination of vaporization. The second inflection point is a local minimum and indicates slowing down of the evaporation rate due to surface accumulation of nanoparticles. Morphology and final precipitation structures of levitated droplets are due to competing mechanisms of particle agglomeration, evaporation, and shape deformation. In this work, we provide a detailed analysis for each process and propose two important timescales for evaporation and agglomeration that determine the final diameter of the structure formed. It is seen that both agglomeration and evaporation timescales are similar functions of acoustic amplitude (sound pressure level), droplet size, viscosity, and density. However, we show that while the agglomeration timescale decreases with initial particle concentration, the evaporation timescale shows the opposite trend. The final normalized diameter can be shown to be dependent solely on the ratio of agglomeration to evaporation timescales for all concentrations and acoustic amplitudes. The structures also exhibit various aspect ratios (bowls, rings, spheroids) which depend on the ratio of the deformation timescale $\left(t_{d e f}\right)$ and the agglomeration timescale $\left(t_{g}\right)$. For $t_{d e f}<t_{g}$, a sharp peak in aspect ratio is seen at low concentrations of nanosilica which separates high aspect ratio structures like rings from the low aspect ratio structures like bowls and spheroids. (C) 2013 American Institute of Physics. [http://dx.doi.org/10.1063/1.4775791]
\end{abstract}

\section{INTRODUCTION}

Acoustic levitation permits the study of droplet dynamics without the effects of surface interactions present in other techniques such as pendant droplet methods. Despite the complexities of the interactions of the acoustic field with the suspended droplet, acoustic levitation ${ }^{1-4}$ provides distinct advantages of controlling morphology of droplets with nanosuspensions post precipitation. ${ }^{5-9}$ Droplet morphology is controlled by vaporization, deformation, and agglomeration of nanoparticles, and therefore, their respective timescales are important to control the final shape. The balance of forces acting on the droplet, such as the acoustic pressure and surface tension, determine the geometry of the levitated droplet. Thus, the morphology of the resultant structure can be controlled by manipulating the amplitude of the levitator and the fluid properties of the precursor nanosuspensions. ${ }^{10-15}$ The interface area in colloidal nanosuspensions is very large even at low particle concentrations. The effects of the presence of this interface have large influence in the properties of the solution even at low concentrations. For example, the viscosity of a nanosilica solution at $2 \%$ concentration can be $40 \%$ higher than that of pure fluid. ${ }^{10-13}$

\footnotetext{
a) All the authors contributed equally to this work.

b) Author to whom correspondence should be addressed. Electronic mail: Ranganathan.Kumar@ucf.edu.
}

Structure formation is a direct consequence of particle agglomeration, the rate at which particles agglomerate and the rate at which the liquid phase in the droplet evaporates determine the final structure size and morphology. ${ }^{16-19}$ Agglomeration of suspended particles is an important field of study which delineates two processes called perikinetics and orthokinetics. Perikinetics deals with agglomeration of suspended particles due to Brownian rotation and diffusion, while orthokinetics arises from shear/velocity gradient within the liquid layers. Bremer et al. ${ }^{16}$ estimated the time scale of macroscopic aggregation which occurs when precipitation is visible. The number of bonds formed and aggregation rate are important for the aggregation process. This led them to define a physical time scale based on orthokinetic sedimentation which did not include arbitrary parameters like visibility of precipitation. Using the agglomeration and evaporation time scales in this paper, we study their effect on structure geometry. The objective is to acoustically levitate a droplet containing nanosilica solutions at different concentrations heated by a $\mathrm{CO}_{2}$ laser. This paper analyzes the effect of concentration on the shape of final structure using high speed imaging and IR thermography. In a first attempt of this kind, we show that the ratio of agglomeration to evaporation time, $\mathrm{t}_{\mathrm{g}} / \mathrm{t}_{\mathrm{e}}$, is used to compare the structure formation at different precursor concentrations and is shown to be related to the diameter ratio. 


\section{EXPERIMENTAL METHODS}

The experimental setup consisted of an ultrasonic levitator (Tec5 ultrasonic levitator, $100 \mathrm{kHz}$ ) to suspend the droplet (Figure 1). ${ }^{19}$ The suspended droplet was heated by a $\mathrm{CO}_{2}$ laser irradiating at a wave length of $10.6 \mu \mathrm{m}$ with a nominal beam diameter of $2 \mathrm{~mm}$. The power of the laser was tuned at $0.75 \mathrm{MW} / \mathrm{m}^{2}$ using a power supply controller. An infrared camera was placed perpendicular to the laser beam to measure the temperature of the droplet. The IR camera (FLIR Silver: calibrated for a range of -5 to $200{ }^{\circ} \mathrm{C}$ with an accuracy of $\pm 1{ }^{\circ} \mathrm{C}$ ) was equipped with a microscopic zoom (FLIR G3-F/2) lens to facilitate $3 \times$ magnification with a working distance of $40 \mathrm{~mm}$. The IR camera was operated at a $100 \mathrm{fps}$ and the recorded images were processed to obtain the temperature data of the droplet during heating. The integration time of the IR camera depends on the temperature range adapted. Most of the experiments were performed with a temperature range of $20-80^{\circ} \mathrm{C}$ which needed an integration time of $1.63 \mathrm{~ms}$. The error in temperature detection due to change in emissivity was found to be $0.03{ }^{\circ} \mathrm{C}$ and hence negligible. A high speed camera (Fastec TSHRMM (Troubleshooter HR Monochrome), with a maximum speed of 16000 frames per second) along with a zoom lens assembly (Navi$\operatorname{tar} 6000)$ was used to capture the physical processes within the droplet during the laser irradiation. This camera was placed at an angle of $30^{\circ}$ with the laser beam as shown in Figure 1. The event was recorded at $500 \mathrm{fps}$, the maximum that could be achieved without losing spatial resolution. The images from high speed camera were used to measure the instantaneous diameter of the droplet with a better time resolution than the IR camera (100 fps).

In our study, nanosilica concentrations ranging from $0.1 \%$ to $7.33 \%$ volume concentration percentage were used. The mean nanoparticle size is of the order of $10 \mathrm{~nm}$. The droplets were generated and deployed to the pressure node of the levitator by a micro needle. For every run of the experiment, the initial diameter of the droplet was maintained to be $500 \mu \mathrm{m}( \pm 30 \mu \mathrm{m})$. After the experiment, the IR and high speed images were analyzed to obtain the temperature and diameter data. The temperature data were obtained by defining a zone of interest around the surface of the drop-

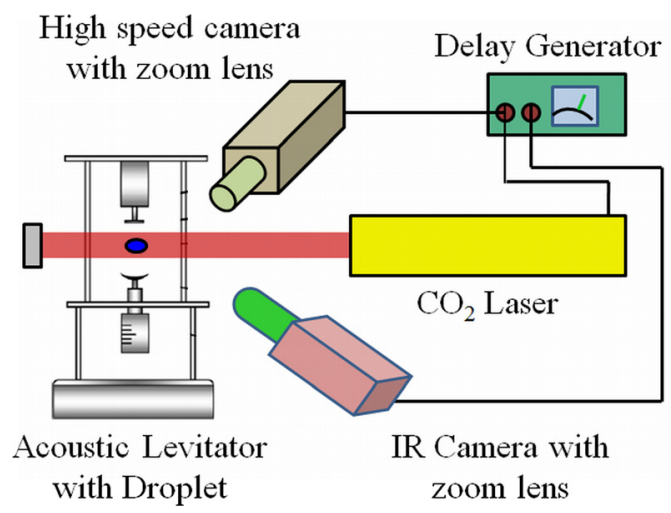

FIG. 1. Experimental setup. Reprinted with permission from (see Ref. 19) S. Basu, A. Saha and R. Kumar, "Criteria for Thermally Induced Atomization and Catastrophic Breakup of Acoustically Levitated Droplet," Int. J Heat Mass Transfer (to be published). Copyright 2013 Elsevier. let in each IR images, and the maximum, average, and standard deviation of the temperature within droplet were calculated. It is important to mention here that the droplet oscillates from side to side with respect to the IR camera axis during the experiment resulting in out of focus images. For aforementioned analysis only those images were considered where the droplet was in the focus plane.

The high speed images were used as evidence of different shape transformations during the heating process. Also these images were used to calculate the diameter of the droplet. The images were analyzed using MOTION MEASURE software. To calculate the instantaneous diameter, an edge around the droplet was defined. An equivalent diameter was calculated from the area under the curve (edge) assuming the droplet to be axisymmetric with respect to the levitator longitudinal axis. The final form of the precipitate was collected on copper grids to perform ex-situ analyses. The samples were analyzed using optical microscope and scanning electron microscopy (SEM). The schematic of the experimental setup is shown in Figure 1. More details about the experimental methods can be found in our previous works. ${ }^{14,15}$

\section{RESULTS AND DISCUSSION}

Radiatively heated levitated functional droplets exhibit three distinct stages namely pure evaporation, agglomeration, and finally structure formation. During the initial stages of the vaporization process, the droplet surface temperature increases due to the monochromatic irradiation of the $\mathrm{CO}_{2}$ laser. The increase in temperature is primarily due to the low vaporization rate and consequently transient heating of the droplet. The temperature front is initially asymmetric (due to directional laser flux) and subsequently advances over the surface (Figure 2). The droplet vaporization rate and temperature homogenization are mainly controlled by the forced convection caused by internal flow recirculation in the liquid phase induced by acoustic streaming (an effect caused by the scattering of the acoustic field due to the presence of the liquid droplet which has a large acoustic impedance compared to the gaseous phase) and droplet rotation about the vertical axis induced by the acoustic torque. Thus, acoustic levitation properties, such as sound pressure level (SPL), have a direct impact on liquid evaporation.

\section{A. Temperature profiles}

The droplet surface temperature profile exhibits two inflection points during the entire evaporation and agglomeration lifecycle (Figs. 3(a) and 4(a)). Initially with continuous heating, the temperature increases and reaches a maximum (first inflection point), which is a function of droplet specific heat, vapor pressure, latent heat, internal recirculation

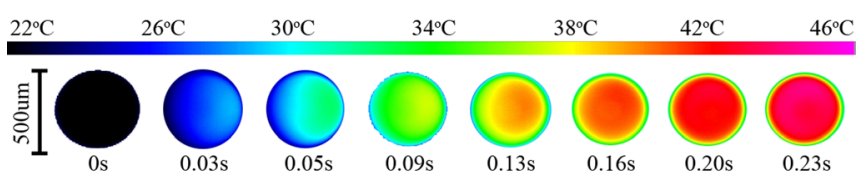

FIG. 2. Infrared images of the droplet temperature increase during early stages of the evaporation phase. 

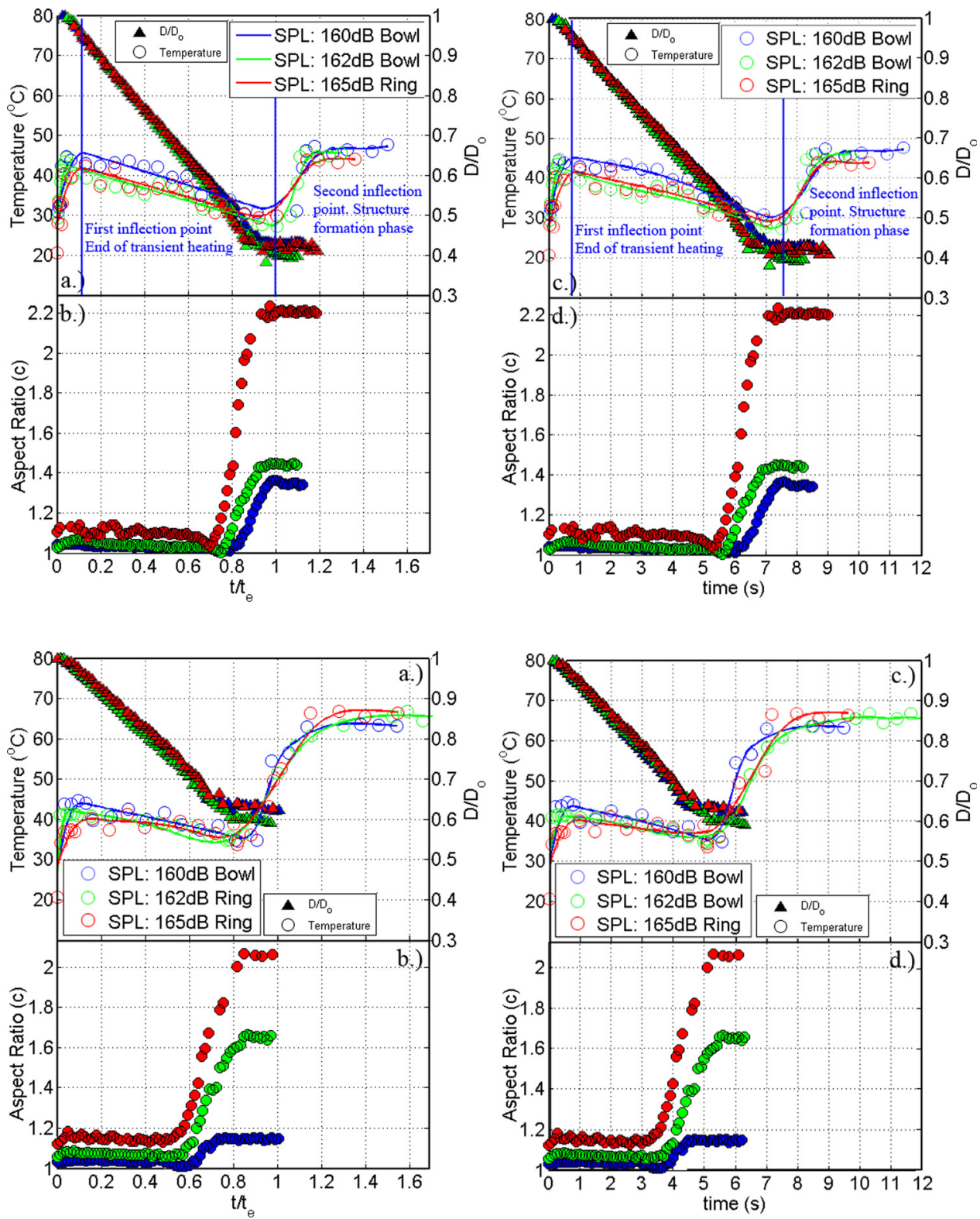

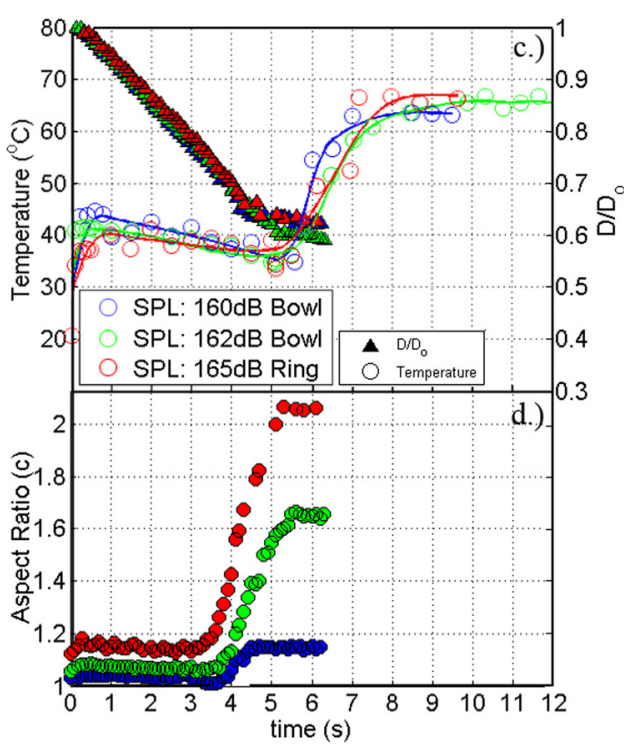

FIG. 3. (a) Surface temperature and D/Do vs. $\mathrm{t} / \mathrm{t}_{\mathrm{e}} \varphi=0.5 \%$; (b) aspect ratio vs. $\mathrm{t} / \mathrm{t}_{\mathrm{e}}$ $\varphi=0.5 \%$. (c) Surface temperature and D/Do vs. time $\varphi=0.5 \%$; (d) aspect ratio vs. time $\varphi=0.5 \%$.
FIG. 4. (a) Surface temperature and D/Do vs. $\mathrm{t} / \mathrm{t}_{\mathrm{e}} \varphi=2 \%$, (b) aspect ratio vs. $\mathrm{t} / \mathrm{t}_{\mathrm{e}}$ $\varphi=2 \%$. (c) Surface temperature and D/Do vs. time $\varphi=2 \%$; (d) aspect ratio vs. time $\varphi=2 \%$. strength, and projected area available for radiative heat transfer. Strong liquid phase recirculation induced by the acoustic streaming generally increases the timescale of the transient temperature rise or, in other words, delays the occurrence of the first inflection point in the temperature profile (Figs. 3(a) and 4(a)). Internal recirculation also aids in reducing the diffusion length (increases the energy transport) and hence constrains the transient temperature rise of a droplet to a lower wet bulb limit. Thus, by tuning the sound pressure level, the magnitude of the acoustic streaming can be adjusted and hence one can control the wet bulb temperature and transient heating timescale. Hence, the maximum temperature in the normalized time domain is higher at an SPL of $160 \mathrm{~dB}$ compared to $165 \mathrm{~dB}$ in the evaporation phase as shown in Figures 3(a) and 4(a). Liquid evaporation is affected by SPL through heat transfer enhancement by virtue of strong recirculation.

Once the droplet attains the wet bulb temperature, vaporization rate becomes maximum (first inflection point of the temperature profile in Fig. 3(a)). However, in radiatively heated droplets (unlike convectively heated droplets), the droplet tends to dissipate heat to the cooler ambient. This leads to a slow decrease in droplet surface temperature. Surface temperature reaches the second inflection point when liquid evaporation rate decreases with increase of particle concentration at the droplet surface. Consequently, the heat absorbed from the laser flux raises the temperature of the porous structure abruptly (second inflection point in Figures 3(a) and 4(a)). The maximum temperature attained by this structure is a weak function of the aspect ratio. Usually, there is a decrease of available projected area for heating as aspect ratio increases with increase in SPL (Figures 3(b) and 4(b)). The maximum temperature of the $\varphi=2 \%$ silica solutions is larger compared to the $\varphi=0.5 \%$ due to larger exposed heating area since rings formed at this particle concentration reorient (from horizontal to vertical plane). In contrast, with the $\varphi=0.5 \%$ cases at 160 and $162 \mathrm{~dB}$, the structure formed at a higher SPL has a larger aspect ratio; therefore, the area exposed to heating is smaller, which yields a lower final 
surface temperature. The diameter reaches a constant value marking the formation of the agglomerated particle structure at different concentrations and different SPL (Figures 2(a) and 3(a)). Aspect ratio also reaches a constant value at the end of the evaporation phase. Figures 3(c) and 4(c) present $\mathrm{D} / \mathrm{D}_{\mathrm{o}}$ and temperature vs. time plots, which clearly show that the total time before structure formation varies between 5 and $8 \mathrm{~s}$. The droplets with higher initial concentration of nanosilica (Fig. 4(c)) exhibits structure formation relatively faster compared to the droplets with low initial concentration (Figure 3(c)). Similarly, Figures 3(d) and 4(d) represent the temporal variation of aspect ratio (c). The sharp increase in aspect ratio coincides with the inflection point of the diameter regression plots (Figures 3(c) and 4(c)).

\section{B. Timescales of vaporization and agglomeration}

As shown in our earlier work, ${ }^{15}$ the timescale for pure evaporation stage is smaller for higher concentration since the amount of solvent to be vaporized is less. Higher solute concentration also suppresses the vapor pressure according to Raoults' law, slowing down the vaporization rate. The total time of the droplet lifecycle (vaporization and agglomeration) is normalized by the evaporation time scale $t_{e}$, which is the time required to evaporate the liquid phase in the droplet. The evaporation timescale is composed of two parts. One part corresponds to the structure formation time $t_{f}$ which is determined by the time taken by the droplet to form rigid structure with no further decrease in droplet diameter. Beyond $t_{f}$, evaporation continues as the liquid phase is still present within the structure although no further surface regression is detected. The volume of this residual liquid is calculated by subtracting the volume at the time of structure formation $\left(V_{f}\right)$ from the initial liquid volume $\left(V_{o}\right)$. Finally, one can write the evaporation time scale $t_{e}$ as

$$
t_{e}=t_{f}+\frac{V_{o}-V_{f}}{\dot{V}_{f}},
$$

where $\dot{V}_{f}$ is the liquid evaporation rate at the instant of structure formation.

\section{Structural morphology}

The nanosilica particles in the droplet are transported by internal recirculation induced by acoustic streaming. As the liquid phase evaporates, the particle concentration near the droplet surface increases and generates a concentration gradient inside the droplet. Solution viscosity increases with particle concentration, ${ }^{10-14}$ thus the radial increase in concentration yields a radial increase in viscosity. The fluid momentum is dissipated in the vicinity of the highly viscous droplet surface. Ultimately, agglomeration is triggered near the surface leading to the formation of a shell shaped structure. The microscopic images show that the particles agglomerate into a semisolid lattice, i.e., a gel morphology. Structure formation is a consequence of the particle agglomeration, and the rate at which the particles agglomerate into a gel is a determining factor on the structure final dimensions. The different structures (Figure 5) exhibit thin to thick shell type feature though the overall shape may resemble a bowl, apple-like spheroid, or a ring. ${ }^{10}$ The final geometry of the structure is not only a function of particle concentration but also of the flow dynamics, evaporation, and agglomeration characteristics.

Evaporation rate governs the rate at which the concentration increases and is a major contributor in determining the structure geometry. Since recirculation velocity (function of both viscosity and sound pressure level) decreases with viscosity, the particle migration is affected by it. Agglomeration occurs when particles collide due to bulk motion in the droplet. This orthokinetic agglomeration mechanism is a hydrodynamic driven process and has no dependence on particle size and is a function of particle concentration and flow shear rate. In short, the rate at which the particles migrate to the droplet surface is a function of viscosity which at any time during the evaporation phase is determined by particle

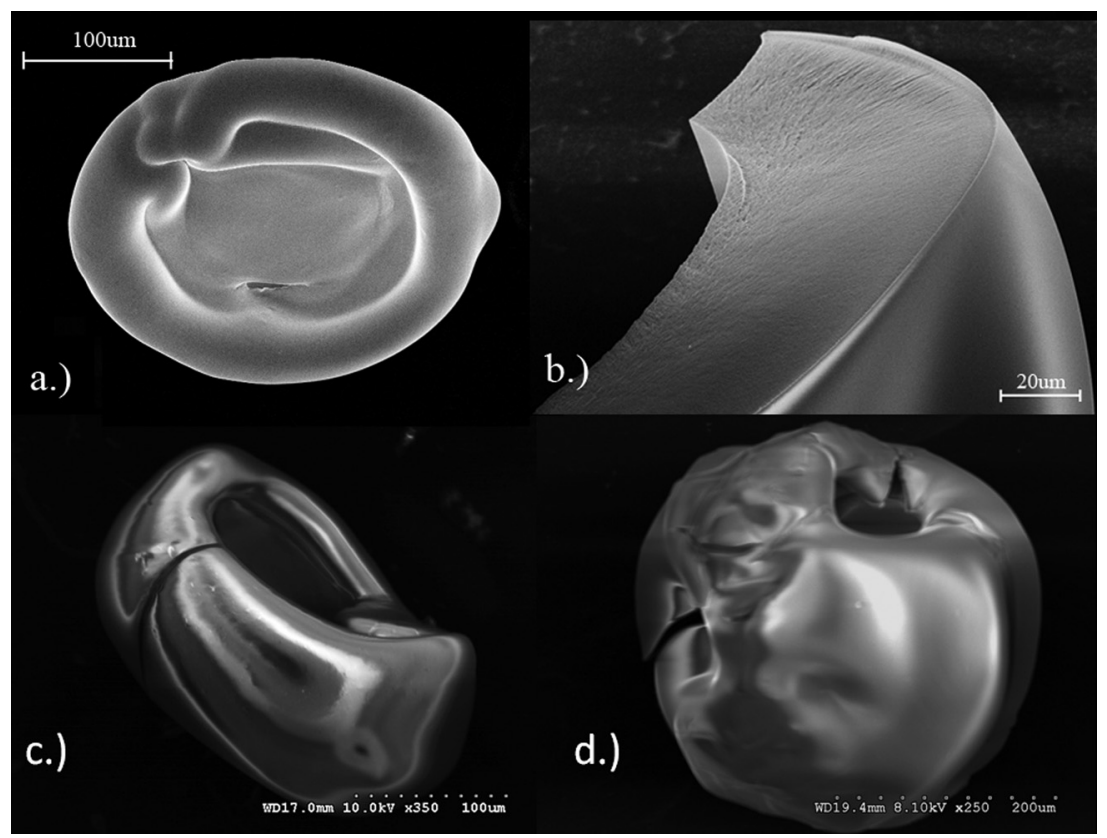

FIG. 5. SEM images of different structure morphologies. (a) $10 \mathrm{~nm}$ silica $\varphi=0.5 \%$ bowl; (b) $10 \mathrm{~nm}$ silica $\varphi=7.33 \%$ spheroid section; (c) $10 \mathrm{~nm}$ silica $\varphi=2 \%$ ring; (d) $10 \mathrm{~nm}$ silica $\varphi=7.33 \%$ spheroid. 
concentration, which in turn, changes with the liquid phase evaporation rate.

\section{Agglomeration timescale}

The effects of evaporation and agglomeration rates are strongly coupled based on the initial concentration and determine the final morphology of the precipitated droplet. The effect of each mechanism, i.e., evaporation rate and agglomeration rate, can be compared by using appropriate time scales. In addition to $\mathrm{t}_{\mathrm{e}}$ in Eq. (1), an orthokinetic time scale may be defined as the time required for particles to agglomerate into a gel.

Bulk agglomeration occurs in other systems such as nanofluid boiling due to strong shear and thermal fields. This has also been previously shown by Bremer et al. ${ }^{16}$ that low shear rates of $0.1 \mathrm{~s}^{-1}$ due to small fluctuations in temperature or velocity can induce substantial agglomeration in nanosilica (20 nm particle size) solutions in quiescent conditions. In our current work, the recirculation velocity induced in the droplet due to acoustics is of the order of $1-10 \mathrm{~cm} / \mathrm{s}$ which corresponds to a shear rate of $20-200 \mathrm{~s}^{-1}$ for a $500 \mu \mathrm{m}$ droplet causing particle bulk agglomeration within the droplet. The shear rate leads to significant particle agglomeration. In previous works by our group, ${ }^{10,11}$ on nanosilica suspensions in pool boiling of water in $0.5 \%-2 \%$ concentrations as in the current experiments, particle agglomeration has been observed due to a combination of perikinetic and orthokinetic agglomeration mechanisms. Particle size measurements were taken using digital light scattering (DLS) technique, and at $0.5 \%$, the $10 \mathrm{~nm}$ particles doubled in size on average due to agglomeration, with the size extending to $\sim 50 \mathrm{~nm}$ in the histogram.

In this study, the agglomeration is dominated by the hydrodynamics and collisions mainly due to the large velocity gradients within the droplet induced by acoustic streaming. The temperature increase also aids in the agglomeration by increasing the particle collision frequency. Agglomeration occurs even in the absence of strong evaporation, i.e., during normal drying of droplets. However, it is to be noted that strong evaporation leads to significant accumulation of particles near the surface which aids in the collision and faster bulk agglomeration. So even though particle growth may not happen due to evaporation induced accumulation, evaporation aids in increasing particle number density which subsequently increases the collision rate, thus enhancing bulk agglomeration. The diffusion coefficient for $10 \mathrm{~nm}$ silica particles is of the order of $2.7 \times 10^{-11} \mathrm{~m}^{2} / \mathrm{s}$. The velocity of droplet regression is $33 \times 10^{-6} \mathrm{~m} / \mathrm{s}$ approximately. Hence, the Peclet number based on droplet diameter is around 600. This high value of Peclet number shows that diffusion is very slow compared with regression of the surface and radial transport due to acoustic induced shear is very fast compared with surface regression.

An agglomeration time scale can then be calculated assuming shear induced particle bulk agglomeration; this time scale is an order of magnitude estimate on agglomeration time. The evaporation and colloidal repulsive forces influence the actual agglomeration time, and the order of magnitude of the timescale remains unaltered, though the exact value may vary. In order to calculate the gel agglomeration scale, ${ }^{16}$ assumptions such as fractal agglomeration and particle monodispersity are taken into account. The time scale for the start of gel formation is defined as ${ }^{16}$

$$
\mathrm{t}_{\mathrm{g}}=\frac{\pi \mathrm{D}}{4 \dot{\gamma}(3-\mathrm{D})}\left(\varphi_{\mathrm{o}}^{-1}-1\right)
$$

where $\mathrm{D}$ is fractal dimensionality, $\varphi_{\mathrm{o}}$ is the initial particle concentration, and $\dot{\gamma}$ is shear rate or the velocity gradient in the radial direction defined as

$$
\dot{\gamma}=\frac{u_{l}}{r_{d}} ; \quad u_{l}=\sqrt{\frac{\mu_{o} \rho_{o}}{\mu_{l} \rho_{l}}} u_{\max },
$$

where $r_{d}$ is the droplet radius and $\mathrm{u}_{l}$ is the liquid phase velocity scale. ${ }^{5}$ Subscripts $o$ and $l$ denote the gas and liquid phases, respectively. $u_{\max }$ is the maximum velocity in the acoustic field, $u_{\max }=M a c_{o} . c_{o}$ is the speed of sound in the gas phase and $\mathrm{Ma}$ is the mach number of the acoustic field related to SPL as given by

$$
S P L=197+20 \log (M a) .
$$

The orthokinetic time scale $\left(t_{g}\right)$ is therefore a function of fractal dimensionality, initial particle concentration, sound pressure level, viscosities of the liquid and gas phase, density of the liquid phase, and droplet size. The observation that particles agglomerate when heated and sheared (due to acoustics) at a high value of Peclet number lends credibility to the usage of the timescale proposed in Eq. (2).

Since the SPL level is strong enough to induce a surface velocity in the liquid phase of the order of $1-20 \mathrm{~cm} / \mathrm{s}$ (see our PIV (Particle Image Velocimetry) measurements in $500 \mu \mathrm{m}$ droplets ${ }^{18}$ ). This recirculation strongly influences the particle transport and shear rate and hence controls the agglomeration in addition to the thermal field created by radiative heating. It can be seen that the agglomeration timescale decreases with increase in liquid phase velocity. The liquid velocity can be augmented in two ways: by decreasing the viscosity of the liquid phase or by increasing SPL. So the agglomeration timescale is considerably shorter for low viscosity fluids subjected to high SPL.

Evaporation timescale on the other hand depends on the initial particle concentration, sound pressure level, and liquid viscosities. High initial particle concentration retards the vaporization rate leading to a longer evaporation timescale. Similarly, high SPL leads to increased recirculation velocity inside the droplet which leads to enhanced vaporization, effectively reducing the diffusion length by almost three times. Low viscosity fluids also lead to strong recirculation that further enhances vaporization.

From Eqs. (2) and (3), $t_{g} \sim \sqrt{\mu_{o}}$, implying that orthokinetic timescale increases with solution viscosity. Evaporation time scale $\left(t_{e}\right)$ is also affected by solution viscosity. Typically, the heat transfer inside the droplet scales with the 
recirculation velocity $\left(\sim u_{l}\right)$ which is affected by solution viscosity. Therefore,

$$
t_{e} \sim \frac{1}{u_{l}} \sim \sqrt{\mu_{o}} .
$$

Change in viscosity therefore has similar effects on $t_{e}$ and $t_{g}$. This suggests that the ratio, $t_{g} / t_{e}$ has a low sensitivity to a change in solution viscosity. Similarly, for a change in SPL

$$
t_{g} \sim \frac{1}{\dot{\gamma}} \sim \frac{1}{u_{l}}
$$

From Eq. (4),

$$
u_{l} \sim u_{\max } \sim M a C_{o} \sim C_{o} e^{(S P L-197) / 20}
$$

Finally

$$
t_{g} \sim \frac{1}{C_{o} e^{(S P L-197) / 20}}
$$

$t_{g}$ decreases by a factor of 1.3 for an increase in SPL from $160 \mathrm{db}$ to $165 \mathrm{db}$. SPL directly influences the recirculation velocity, which affects the evaporation scale by reducing the diffusion distance by a factor of 2 . The evaporation timescale thus reduces with increase in acoustic streaming in a similar way as the orthokinetic timescale. Thus, the ratio $t_{g} / t_{e}$ has relatively lower sensitivity to SPL.

Finally, initial particle concentration also has a large effect on both $t_{g}$ and $t_{e}$. From Eq. (2), $t_{g}$ scales with particle concentration as $t_{g} \sim \frac{1}{\varphi}$ which signifies that orthokinetic time scale decreases with particle concentration (since the distance between particles decreases with particle concentration). The frequency of particle collisions increases, causing an increase in agglomeration rate. On the contrary, evaporation time calculated based on the initial volume of the liquid in the droplet (in the absence of agglomeration) should decrease with increase in initial particle concentration mainly due to the resistance offered by the surface accumulation of the nanoparticles

$$
\begin{aligned}
t_{e} & \sim \frac{1}{m_{\text {vaporization }}} \sim\left[\ln \left(1+B_{m}\right)\right]^{-1} \\
& =1 /\left[\ln \left(\frac{1}{1-Y_{v, s}}\right)\right] ; \quad Y_{v, s} \sim p_{v, s} \sim\left(\varphi p_{\text {sat }}\right),
\end{aligned}
$$

where $Y_{v, s}$ and $p_{v, s}$ are the vapor mass fraction and vapor pressure at the droplet surface and $\varphi$ signifies initial concentration of the nanoparticles. Thus, $t_{e}$ will increase with increase in initial particle concentration. This functional dependence of $\mathrm{t}_{\mathrm{e}}$ on initial concentration is opposite to the orthokinetic timescale. Hence, $t_{g} / t_{e}$ ratio will exhibit high sensitivity to initial concentration. $t_{g} / t_{e}$ will decrease with increase in initial concentration (Figure 6), and the normalized diameter, $\mathrm{D} / \mathrm{D}_{0}$, of the final structure scales with the inverse power of $t_{g} / t_{e}$. For small $t_{g} / t_{e}$, particles begin agglomerating faster near the droplet surface even when the diameter of the droplet has not reduced appreciably mainly due to the time required for evaporation being longer than the time
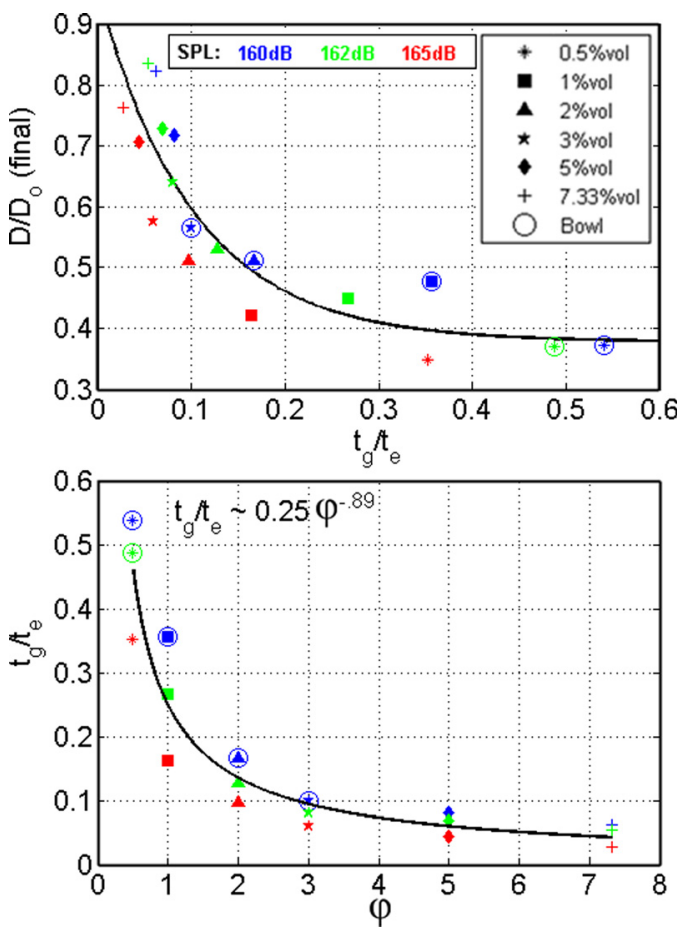

FIG. 6. Final D/Do with respect to the ratio of orthokinetic time scale $\left(t_{g}\right)$ and liquid phase evaporation time $\left(\mathrm{t}_{\mathrm{e}}\right)$.

required for particle agglomeration. At $t g / t e \sim 1$, agglomeration occurs after significant reduction in droplet size due to evaporation. At high concentrations, $\mathrm{tg} / \mathrm{te}$ is generally low. The magnitudes of the different time scales for the different particle concentrations have been compared in Table I.

\section{E. Deformation timescale}

The final structure formed by the droplets exhibit a variety of morphological shapes varying from bowls to rings. These structures can be differentiated from each other based on their aspect ratios. Hence, it is clearly understood that the

TABLE I. Time scales for different processes.

\begin{tabular}{lrrrr}
\hline \hline$\varphi(\%$ vol. $)$ & SPL $(\mathrm{dB})$ & $\mathrm{t}_{\mathrm{e}}(\mathrm{s})$ Eq. $(1)$ & $\mathrm{t}_{\mathrm{g}}(\mathrm{s}) \mathrm{Eq} .(2)$ & $\mathrm{t}_{\mathrm{def}}(\mathrm{s}) \mathrm{Eq} .(11)$ \\
\hline 0.5 & 160 & 10.379 & 5.607 & $6.38 \times 10^{-4}$ \\
& 162 & 9.678 & 4.723 & $5.26 \times 10^{-4}$ \\
& 165 & 8.870 & 3.125 & $3.56 \times 10^{-4}$ \\
1.00 & 160 & 10.075 & 3.597 & $6.30 \times 10^{-4}$ \\
& 162 & 8.471 & 2.264 & $5.14 \times 10^{-4}$ \\
& 165 & 7.603 & 1.244 & $3.57 \times 10^{-4}$ \\
2.00 & 160 & 12.085 & 2.017 & $7.09 \times 10^{-4}$ \\
& 162 & 11.040 & 1.409 & $5.60 \times 10^{-4}$ \\
3.00 & 165 & 9.473 & 0.914 & $3.59 \times 10^{-4}$ \\
& 160 & 10.658 & 1.062 & $6.46 \times 10^{-4}$ \\
& 162 & 9.433 & 0.761 & $5.00 \times 10^{-4}$ \\
5.00 & 165 & 8.151 & 0.489 & $3.02 \times 10^{-4}$ \\
& 160 & 10.272 & 0.837 & $6.59 \times 10^{-4}$ \\
& 162 & 9.562 & 0.656 & $4.97 \times 10^{-4}$ \\
& 165 & 8.766 & 0.386 & $3.46 \times 10^{-4}$ \\
& 160 & 14.430 & 0.886 & $6.53 \times 10^{-4}$ \\
& 162 & 12.414 & 0.661 & $5.16 \times 10^{-4}$ \\
& 165 & 11.449 & 0.318 & $3.34 \times 10^{-4}$ \\
\hline \hline
\end{tabular}




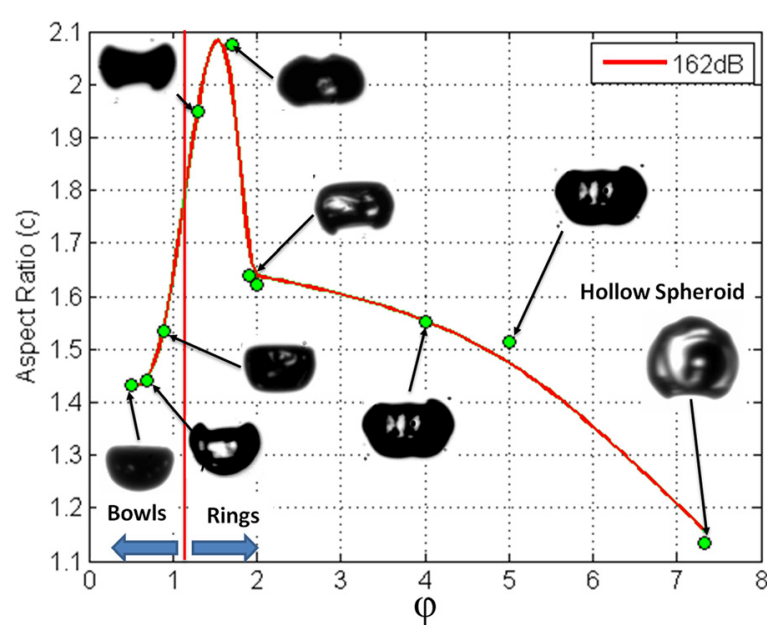

FIG. 7. Final aspect ratio of the agglomerated structure with respect to initial particle concentration.

droplets deform considerably due to the acoustic force while during the vaporization and agglomeration process. For a droplet at equilibrium, the acoustic pressure force acting on it has to be balanced by surface tension forces. Acoustic pressure is a function of SPL and the magnitude of Laplace pressure is inversely proportional to the interface curvature which in turn is inversely proportional to aspect ratio. The Laplace pressure and rms value of the acoustic pressure are of similar magnitude prior to precipitation (inflection point of the $\mathrm{D} / \mathrm{D}_{0}$ plot, Figure 3 ).

The aspect ratio should ideally increase with increase in SPL in order to balance the additional acoustic pressure force acting on the droplet surface that may lead to droplet disintegration. As aspect ratio increases, the droplet minor axis length decreases (considering the droplet to be elliptical). The temporal variation of the minor axis of the droplet can be scaled as (neglecting surface tension and viscosity)

$$
m_{i} \frac{d^{2} y}{d t^{2}} \sim P_{\text {Acoustic }} A_{\text {droplet }}
$$

where $y$ is the displacement of the droplet pole in the acoustic field axial direction, corresponding to a variation in droplet minor axis length, $\Delta y=\Delta b$. The ideal time scale for droplet deformation $\left(t_{d e f}\right)$ is derived by integrating Eq. (7), yielding

$$
t_{d e f} \equiv\left[\frac{y * m_{i}}{p_{B} A_{d}}\right]^{1 / 2}
$$

The relative magnitudes of the orthokinetic scale $\left(t_{g}\right)$ and deformation time scale $\left(t_{d e f}\right)$ determine the final structure aspect ratio. If $t_{d e f}<t_{g}$, particle agglomeration occurs as the droplet has been already deformed substantially by the acoustic field. At this instant, the droplet aspect ratio is already large. When $t_{d e f}>t_{g}$, the increase in aspect ratio requires a longer time than the agglomeration process. Consequently, the particles agglomerate while the aspect ratio is still small. Once agglomeration is triggered, the droplet tends to remain undeformed since the surface rigidity is enhanced by the particulate shell (Figure 7). For $\varphi<2 \%$, the orthoki- netic timescale is comparatively large. This is reflected by the fact that the droplet deforms substantially before agglomeration is triggered resulting in a large aspect ratio structure. On the other hand, for $\varphi>2 \%$, the orthokinetic timescale is low which leads to faster agglomeration compared to deformation. This yields low aspect ratio structures. Therefore, an inflection point is created near $\varphi \sim 2 \%$ which separates high and low aspect ratio structures.

\section{CONCLUSIONS}

In summary, this work shows that there are competing timescales for the vaporization, deformation, and agglomeration processes. The normalized diameter is inversely proportional to the ratio of agglomeration to evaporation timescales for all concentrations and acoustic amplitude. It is seen that both agglomeration and evaporation timescales are similar functions of acoustic amplitude (sound pressure level), droplet size, viscosity, and density. However, we showed that while the agglomeration timescale decreases with initial particle concentration, the evaporation timescale shows the opposite trend. The final normalized diameter hence can be shown to be dependent solely on the ratio of agglomeration to evaporation timescales for all concentrations and acoustic amplitudes. The structures also exhibit various aspect ratios (bowls, rings, and spheroids) which depends on the ratio of the deformation timescale $\left(\mathrm{t}_{\mathrm{def}}\right)$ and the agglomeration timescale $\left(t_{g}\right)$. A sharp peak in aspect ratio is seen at $\sim 2 \%$ concentration. For $t_{\text {def }}<t_{g}$, a sharp peak in aspect ratio is seen at low concentrations of nanosilica which separates high aspect ratio structures like rings from the low aspect ratio structures like bowls and spheroids.

${ }^{1}$ W. J. Xie and B. Wei, Appl. Phys. Lett. 90, 204104-1-204104-3 (2007).

${ }^{2}$ W. J. Xie, C. D. Cao, Y. J. Lü, and B. Wei, Phys. Rev. Lett. 89, 104304 (2002).

${ }^{3}$ W. J. Xie and B. Wei, Phys. Rev. E 66, 026605 (2002).

${ }^{4}$ R. Vehring, Pharm. Res. 25(5), 999-1022 (2008).

${ }^{5}$ A. Yarin, M. Pfaffenlehner, and C. Tropea, J. Fluid Mech. 356, 65-91 (1998).

${ }^{6}$ Y. Tian and R. Apfel, J. Aerosol Sci. 27, 721 (1996).

${ }^{7}$ A. Yarin, G. Brenn, O. Kastner, D. Rensink, and C. Tropea, J. Fluid Mech. 399, 151-204 (1999).

${ }^{8}$ H. Zhao, S. S. Sadhal, and E. H. Trinh, J. Acoust. Soc. Am. 106, 32893295 (1999).

${ }^{9}$ E. G. Lierke, Acta. Acust. Acust. 88, 206-217 (2002).

${ }^{10}$ D. Milanova and R. Kumar, Appl. Phys. Lett. 87, 233107 (2005).

${ }^{11}$ R. Kumar and D. Milanova, "Dispersion and surface characteristics of nano-oxide suspensions,” Ann. N.Y. Acad. Sci. 1161, 472-483 (2009).

${ }^{12}$ D. Milanova and R. Kumar, J. Heat Transfer 130, 042401 (2008).

${ }^{13}$ P. Vassallo, R. Kumar, and D’Amico, Int. J. Heat Mass Transfer 47, 407 (2004).

${ }^{14}$ R. Kumar, E. Tijerino, A. Saha, and S. Basu, Appl. Phys. Lett. 97, 123106 (2010).

${ }^{15}$ A. Saha, S. Basu, C. Suryanarayana, and R. Kumar, Int. J. Heat Mass Transfer 53, 5663 (2010).

${ }^{16}$ L. G. B. Bremer, P. Walstra, and T. van Vliet, Colloids Surf. 99, 121-127 (1995).

${ }^{17}$ F. Iskandar, L. Gordon, and K. Okuyama, J. Colloid Interface Sci. 265, 296-303 (2003).

${ }^{18}$ A. Saha, S. Basu, and R. Kumar, Phys. Lett. A 376(45), 3185-3191 (2012).

${ }^{19}$ S. Basu, A. Saha, and R. Kumar, "Criteria for thermally induced atomization and catastrophic breakup of acoustically levitated droplet," Int. J. Heat Mass Transfer, doi: 10.1016/j.ijheatmasstransfer.2012.12.040. 